\title{
EL EXTRAÑO CASO DEL VERBO BÚLGARO. UNA LECTURA DESDE LA TEORÍA VECTORIAL DE GUILLERMO ROJO
}

\author{
TANia Dimitrova LÁleVa
}

\section{RESUMEN}

Este artículo estudia las formas verbales búlgaras compuestas con al menos un participio en $-l$. Estas formas impregnan todo el sistema del verbo búlgaro y clarificar su clasificación y uso continúa siendo una tarea pendiente. La presente investigación parte de los principios de organización propuestos para el sistema temporal por Benveniste y Weinrich, y busca el lugar que ocupan las formas con participios en - $l$ en el sistema temporal búlgaro, contrastándolo con el sistema temporal espanol mediante la aplicación de la teoría vectorial de Guillermo Rojo.

Palabras clave: verbo búlgaro, sistema temporal, participio en -l, teoría vectorial.

\section{ABSTRACT}

This article studies the Bulgarian verbal forms composed with at least a participle in $-l$. These forms impregnate all the system of the Bulgarian verb and to clarify its classification and use continues being a pending task. Our investigation relies in the organizational principles proposed by Benveniste and Weinrich for the temporal system and looks for the place that the forms with participles in- $l$ in the Bulgarian temporal system occupy in such system, contrasting it with the Spanish temporal system and applying the vectorial theory of G. Rojo.

Key Words: Bulgarian Verb, Temporal System, Participle in -l, Vectorial Theory.

«El verbo es el elefante en el reino de la gramática búlgara»

A. T. BALAN

\section{INTRODUCCIÓN}

El búlgaro es una lengua eslava que hoy no comparte una serie de características lingüísticas importantes con el resto de lenguas de su grupo. Una de las más llamativas es la existencia de un sistema verbal peculiar que, por un lado, conserva el sistema temporal del antiguo eslavo con una continuidad formal indiscutible y, por otro, desarrolla una serie de formas verbales cuyo rasgo común es el participio en -l. Mantener las 
diferencias temporales y crear un paradigma verbal paralelo, funcional y semánticamente definido sobre el participio en - $l$ es un hecho lingüístico de enorme interés. Nuestra investigación pretende presentar y defender una teoría coherente sobre el sistema temporal búlgaro y el lugar que ocupan en este las formas con participio en $-l$ aplicando la teoría vectorial de Guillermo Rojo y contrastándolo con el sistema temporal español.

Según el investigador búlgaro P. Pašov 1965, p. 52, cada verbo búlgaro tendría unas 1500 formas. Esta abundancia tan inusitada se debe en gran medida a las formas compuestas con participio en $-l$. Relacionar forma, significado y uso fue una de las primeras tareas con la que se enfrentaron los estudiosos del idioma búlgaro, pero que difícilmente podríamos considerar terminada. Hasta la fecha, en la lingüística búlgara no existe unanimidad acerca del sistema verbal búlgaro y del lugar que ocupan en este los paradigmas basados en participios en $-l$. A pesar de las numerosas investigaciones podemos afirmar que siguen sin aclarar casi todos los problemas relacionados con estas formas compuestas:

- el inventario de formas con valor morfológico;

- número y composición de los paradigmas en los que participan;

- su valor semántico;

- su uso y su lugar en el sistema categorial del verbo búlgaro.

Queda claro que hay que buscar un nuevo punto de vista, un nuevo enfoque que nos permita comprender mejor las formas. Si la controversia afecta tanto a su número como a su significado, lo más probable es que nos encontremos ante procesos vivos que no han dejado de estar activos en el último siglo, y que, por tanto, nos encontremos ante un cambio lingüístico importante que, como creo, todavía sigue en marcha.

\section{Delimitación del objeto de ESTUdio}

Antes que nada conviene determinar los conjuntos de formas que vamos a tratar. Nuestro punto de partida ha de ser lo suficientemente amplio como para poder situar las formas compuestas con participio en - $l$ dentro del complicado sistema temporal y modal del verbo búlgaro.

En mi trabajo parto de los siguientes tres grandes grupos de formas verbales:

- GRUPO 1. Las formas con participio en - $l$, todas antiguas, son parte de sus paradigmas. Tradicionalmente se definen como «tiempos resultativos» y entran en el indicativo. Son parte del sistema temporal básico de la lengua búlgara. 
- Grupo 2. Grupo nuevo que abarca los paradigmas surgidos en torno al pretérito perfecto. Las formas compuestas con participio en $-l$ son las únicas posibles en estos paradigmas nuevos.

- GRUPO 3. También nuevo, con rasgo común formal šte(da). Como en el caso del grupo tradicional, las formas en $-l$ ocupan solo parte del paradigma. Por falta de espacio aquí, presentaré los resultados del estudio de este grupo en otro trabajo.

\subsection{Formas del grupo 1}

Son parte del paradigma temporal básico del verbo búlgaro.

$\begin{array}{ll}\begin{array}{l}\text { Presente } \\ \text { Perfecto }\end{array} & \begin{array}{l}\text { piša, piše } \\ \text { pisal săm, pisal e }\end{array} \\ \begin{array}{l}\text { Futuro } \\ \text { Futuro Perfecto }\end{array} & \begin{array}{l}\text { šte piša, šte piše } \\ \text { šte săm pisal, šte e pisal }\end{array} \\ \begin{array}{l}\text { Aoristo } \\ \text { Imperfecto }\end{array} & \begin{array}{l}\text { pisah, pisa } \\ \text { pišeh, pišeše } \\ \text { Pluscuamperfecto }\end{array} \\ \begin{array}{l}\text { Futuro Pretérito } \\ \text { Futuro Pretérito Perfecto } \text { pisal }\end{array} & \begin{array}{l}\text { štjah da piša, šteše da piše } \\ \text { štjah da săm pisal, šteše da e pisal }\end{array}\end{array}$

Como vemos, los tiempos van emparejados. Al lado del presente, futuro y futuro pretérito, encontramos un tiempo compuesto con participio en $-l$ y verbo auxiliar en el tiempo presente, futuro y futuro pretérito según corresponda. Frente al imperfecto y aoristo, por falta de diferencia semántica y formal entre el aoristo y el imperfecto en el auxiliar búlgaro encontramos una única forma compuesta, el pluscuamperfecto $^{2}$. Las formas, salvo el futuro, son heredadas del búlgaro antiguo. Lo que aquí interesa es que las formas compuestas en $-l$ constituyen un grupo bien definido formal y semánticamente. Presentan el verbo auxiliar en el tiempo parejo (presente săm, futuro šte săm, pasado bjah, futuro pretérito štjah da săm) más el participio de aoristo. El significado principal está en relación directa con la forma. La acción marcada por

\footnotetext{
${ }^{1}$ En este trabajo los tiempos se ejemplifican mediante su $1 .^{\underline{a}}$ y/o $3 .^{\underline{a}}$ persona en sg.

${ }^{2}$ Las formas be y beše solo aparentan marcar una diferencia entre el aoristo y el pretérito imperfecto. En realidad proceden de uno de los dos paradigmas del antiguo aoristo ( $\mathbf{6} \mathbf{k} \mathbf{X}^{\mathbf{h}} \mathbf{h}$, $\mathbf{K} \mathbf{k})$, que por su vocal temática - $\mathbf{-}$ - resultaba apropiado para desempeñar también las funcio-

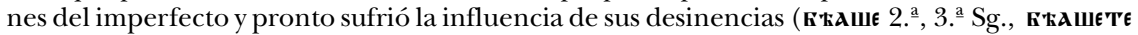

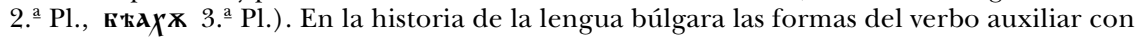
desinencias de aoristo y de imperfecto nunca se separaron semánticamente. Hoy se detecta una cierta preferencia hacia bě̌e en ambas funciones.
} 
el participio como anterior (pisal), se actualiza por el auxiliar que la sitúa en relación con el tiempo marcado por su propia forma temporal (săm, šte săm, bjah, štjah da săm). Puede tratarse, en efecto, como habitualmente se señala, de resultados de la acción, presentes en el tiempo del auxiliar, pero este sería solo uno de los significados adicionales de las formas que no afecta en nada su valor temporal.

\title{
2.2. Formas del grupo 2
}

Contienen por lo menos un participio en $-l$. Desde el punto de vista formal y semántico se distinguen claramente los siguientes conjuntos:

2.2.1. Formalmente derivan de los tiempos pretéritos del grupo 1 . El procedimiento de formación es parecido al aplicado para las formas en $-l$ propias del grupo 1, pero el auxiliar nuevo de la forma resultante está siempre en presente. El participio procede de la «forma finita de pretérito» (pisah > pisal, pišeh > pišel, bjah > bil, štjah > štjal)

Aoristo
Imperfecto
Pluscuamperfecto
Futuro Pretérito
Futuro Pretérito Perfecto

pisal săm, pisal e pišel săm, pišel e bil săm pisal, bil e pisal štjal săm da piša, štjal e da piše štjal săm da săm pisal, štjal e da e pisal

Como se puede apreciar, el aoristo coincide con el perfecto del grupo 1. La coincidencia es formal e histórica, pero también es semántica. Podemos pensar en un perfecto que se convierte en tiempo principal de pasado y crea su propio grupo de formas temporales mediante un procedimiento ya experimentado.

2.2.2. Presenta las mismas formas, pero pierde el auxiliar en $3^{\underline{a}}$ persona.
Aoristo
Imperfecto
Pluscuamperfecto
Futuro Pretérito
Futuro Pretérito Perfecto

\author{
pisal săm, pisal \\ pišel săm, pišel \\ bil săm pisal, bil pisal \\ štjal săm da piša, štjal da piše \\ štjal săm da săm pisal, štjal da e pisal
}

A primera vista el cambio parece insignificante. En efecto, la pérdida de $e$, sa en $3^{\underline{a}}$ persona, la menos marcada, y auxiliar conservado en las demás personas es el desarrollo interno lógico del paradigma. Pero la cosa cambia si observamos el uso real de las formas. Es la $3^{\underline{a}}$ la única per- 
sona en uso en el plano de una historia distante, que obedece a la perspectiva de no participación. En este sentido la falta del auxiliar -que como hemos visto está siempre en presente- rompe la relación de las formas con el plano actual, las deja caracterizadas solo por el participio en $-l$, y con ello las convierte en las más propicias para gramaticalizarse como formas para el plano no coetáneo no participado. Son las formas obligatorias en textos históricos (salvo los de historia reciente), cuentos populares, fabulas y leyendas.

El paradigma completo, las tres personas, ofrece al hablante la posibilidad de enajenar la acción, de marcarla como distante, «no testimonial», lo que cubriría parte del uso, siempre opcional en la lengua búlgara, conocido como «renarrativo». No excluye a los participantes en la comunicación y sitúa la acción en el "plano del pasado coetáneo al hablante». En este plano la acción enajenada sigue en su ámbito temporal, no hay transposición temporal, y lo que marca la forma es la retransmisión de una información (visión) ajena sobre una acción, sin importar que el hablante la haya presenciado, o no, incluso sin importar que la haya protagonizado.

Las mismas formas se emplean también en el «plano actual». En este caso su uso no se corresponde con su valor temporal. Podemos pensar en un proceso de «dislocación» que, como es de esperar, les aporta significados adicionales.

2.2.3. Derivan del grupo 2.2 con la ampliación directa de las formas con el participio en $-l b i l^{3}$. Como resultado, estas formas compuestas presentan por lo menos dos participios en $-l$, uno de los cuales es siempre $b i l$, o sea, el participio de aoristo en $-l$ del verbo auxiliar.

Aoristo
Imperfecto
Pluscuamperfecto
Futuro Pretérito
Futuro Pretérito Perfecto

Aoristo

Imperfecto

Pluscuamperfecto

Futuro Pretérito Perfecto

\author{
pisal săm bil, pisal bil \\ pišel săm bil, pišel bil \\ se emplean las formas del grupo 2.2. \\ štjal săm bil da piša, štjal bil da piše \\ štjal săm bil da săm pisal, štjal bil da e pisal
}

Creo importante subrayar que históricamente estas son las formas más recientes. Que nunca aparecen con auxiliar conservado en $3^{\underline{a}}$ persona y no hay razón alguna que exija derivarlas de las formas del grupo 2.1 para tener que postular después una pérdida inmediata del auxiliar en las formas nuevas tipo pišel $e>$ pišel $e$ bil $>$ pišel bil. Una transforma-

\footnotetext{
${ }^{3}$ A pesar de Kucarov 1999 y Gerdžikov 2003, quienes creen que se derivan del que aquí presentamos como grupo 2.1. con la repetición de la misma técnica, que esta vez se aplicaría sobre el nuevo auxiliar săm, siempre en presente del grupo 2.1 (săm > bil e > bil).
} 
ción mucho más natural sería pišel > pišel bil, además, como pretende demostrar la investigación, el modo aquí propuesto de derivar las formas de este grupo se corresponde mucho mejor con su significado de incredulidad y/o irrealidad que les asigna el hablante.

\section{Consideraciones PREVIAS}

El examen del inventario formal de los nuevos paradigmas del verbo búlgaro demuestra sin lugar a dudas que se trata del desarrollo y explotación de los dos modelos analíticos de los que previamente disponía el sistema. Bien establecido el primero - el de los tiempos perfectos considerados «resultativos» (auxiliar + participio en $-l$ )- desde la época del eslavo antiguo, y en proceso de desarrollo el segundo -el de los tiempos futuros (šte[da] + verbo conjugado) - en el búlgaro medieval, su aplicación reiterada sobre el material lingüístico ha creado nuevas formas compuestas con nuevos significados, o sea, nuevos paradigmas gramaticales.

Centrándonos en el grupo 2, que es el más estructurado, observamos que acoge los tres paradigmas conflictivos que los gramáticos búlgaros han ido denominando «modos», «modo de expresión» o modus constituyentes de una hipercategoría. Así nos encontramos con: «modo renarrativo (pišel)», «modo conclusivo (pišel e)», también modus-modus (dicendi) inveritativus, modus (dicendi) conclusivus y modus (dicendi) renarrativus-, que junto con las formas del grupo 1 -modus (dicendi) testimonialis- formarían, según Gerdžikov 2003, una hipercategoría nueva basada en dos oposiciones, la renarratividad y la subjetividad. Las formas del grupo 2.3 tradicionalmente se definirían como «renarrativas fuertes» o «reforzadas» (inveritativas, según la terminología de Gerdžikov). Todas las formas del grupo 2 podrían estar repartidas de manera totalmente diferente (Stankov 1967, Penčev 1967, Aronson 1967) dentro del indicativo, que presentaría entonces dos grandes series, renarrativa y directa, la última con dos subgrupos: testimonial y neutral, situándose las formas del grupo 2.1 entre las neutrales junto con el presente.

Lo que no ha suscitado ninguna duda hasta la fecha es la importancia primaria de la «renarratividad». Todos los autores han aceptado a priori que el significado central de las formas tipo pišel desde el primer momento ha sido la retransmisión de enunciado ajeno. La existencia de una categoría paralela en la lengua turca reforzaba la convicción de que se trataba de un fenómeno lingüístico bastante nuevo, ajeno al desarrollo de las lenguas eslavas e impulsado de fuera en una época más o menos reciente, llegándose incluso a situar su inicio en el siglo XVII.

No obstante, datos procedentes de textos relacionados con los siglos XII-XIV no corroboran la aparición tardía de las formas. Se trata de uno de los mayores cambios en el sistema de la lengua búlgara, el más 
destacable junto con el analitismo nominal, que, como todo cambio lingüístico, pasa por etapas diferentes y no tiene por qué limitarse a un impulso único.

Hasta la fecha ha sido muy prolija la investigación sobre las coincidencias y las divergencias entre las formas búlgaras definidas aquí como grupo 2 y la categoría gramatical turca considerada su modelo. Se ha mencionado también el substrato protobúlgaro que actuaría en la misma dirección ${ }^{4}$. Lo que no se ha hecho es situar las influencias externas en el fondo de las tendencias inherentes al desarrollo del verbo eslavo, que sería una causa interna derivada de la propia estructura de la lengua. Creo que esta causa no ha sido marginal, sino que ha proporcionado las condiciones imprescindibles para el posterior desarrollo del verbo búlgaro. La gran diferencia entre el sistema verbal contemporáneo del resto de las lenguas eslavas y el búlgaro no puede disimular el hecho de que el antiguo sistema no está conservado en ninguno de los idiomas. Sorprendentemente, donde mejor se conserva es en la lengua búlgara. Algo que no quiere decir que le hayan sido ajenos los procesos que afectaron a las demás lenguas de su grupo. Un primer acercamiento a la gramática histórica del ruso, por ejemplo, arroja luz nueva sobre el fenómeno. El perfecto ha pasado a desempeñar las funciones del imperfecto, primero, y del aoristo, después. Además, en la $3^{\underline{a}}$ persona el

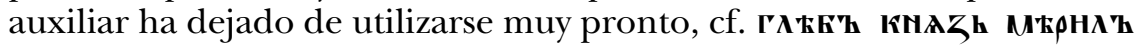
Mope no se A̧ or en la inscripción de T'mutarakan' de 1068. La gramática histórica rusa considera que el perfecto tenía la oportunidad de convertirse en pasado único por su relación doble: con el presente y la «imperfectividad» mediante el auxiliar, y con el pasado y la «perfectividad» gracias al participio en $-l$. La pérdida del auxiliar en todas las personas es más reciente y supone su situación definitiva en el plano del pasado.

También el pluscuamperfecto ruso antes de desaparecer de esta lengua ha cambiado y presenta formas muy parecidas a las búlgaras como podemos ver en los ejemplos citados por Ivanov 1983, p. 352, de textos de los siglos XIII-XIV, cf. "prelstil" mja esi byl" dijavole; šel esi byl" na staryja» (Crónica Lavrentieva); y más a menudo con pérdida del auxiliar en presente «byli na sude iskali kolokola» (Privilegio de Smolensk 1284); «a čto byl" otjal brat tvoi» (Privilegio de Novgorod 1270), «ne leniva mja byl" stvoril» (Crónica Lavrentieva) etc.; y de dialectos rusos contemporáneos: «kazaki byli pošli da opjat' vernulis'», «zemlja byla vysohla da opjat' promokla». Las formas rusas muestran la pérdida paulatina del auxiliar, que en los dialectos falta del todo.

Me parece especialmente interesante la idea de F. Buslaev, sostenida también por P. Černix, según la cual las formas žil-byl, žili-byli que

\footnotetext{
${ }^{4}$ Sobre la historia de las investigaciones dedicadas a la «categoría de renarratividad» se puede consultar Kucarov 1979, Kucarov 1999, Gerdžikov 2003.
} 
encontramos en el principio de los cuentos populares rusos son herederas de este pluscuamperfecto cambiado. Quiero llamar la atención sobre la coincidencia total entre esta fórmula y una de las fórmulas habituales en el cuento popular búlgaro, živjal bil, considerada «renarrativa».

El serbio y el croata, idiomas eslavos meridionales más cercanos al búlgaro que el ruso, también muestran una expansión clara del perfecto que sustituye progresivamente a los tiempos del pasado simples y, lo que parece bastante llamativo, en $3^{\underline{a}}$ persona pierde el auxiliar sobre todo en funciones de pretérito. También el pluscuamperfecto (en indudable recesión) muestra, junto con las antiguas, formas nuevas idénticas a las búlgaras del grupo 2, cf. bejah pevao / bio sam pevao (formas vieja y nueva de pluscuamperfecto serbio) como bjah pjal/ bil săm pjal (pluscuamperfecto del grupo 1 y pluscuamperfecto del grupo 2 en búlgaro).

En parte de las lenguas eslavas el perfecto, y también el pluscuamperfecto han ido sustituyendo al resto de los tiempos del pasado; en realidad, han ido convirtiéndose en formas de pasado a expensas del resto. Podemos entonces hablar de una tendencia fuerte que abarcó a todas las lenguas eslavas en una época, al parecer anterior al siglo XIV. No hay razón alguna para pensar que el búlgaro resultó inmune, que se trataba de algo que no lo afectó en absoluto. El perfecto ha creado en este idioma paradigmas a su imagen y semejanza que cubren prácticamente todo el espacio del pasado, parte de estos con formas en $3^{\underline{a}}$ persona que han ido perdiendo su auxiliar de manera paulatina.

Creo que la tendencia común eslava que reforzaba el uso del perfecto y su grupo fue el caldo de cultivo que permitió el desarrollo «exótico» búlgaro. Sus formas aparecen en función de aoristo ya por el siglo XIV, tal y como muestran los ejemplos de Zlatanova 1986, p. 200:

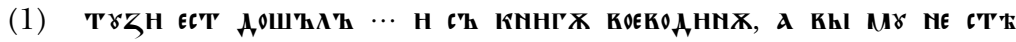

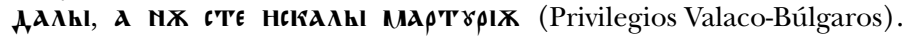

Formas tipo bil săm pisal (análogas a las rusas, serbocroatas, etc.) encontramos en los textos búlgaros desde el siglo xv, Zlatanova 1986, pp. 200 y s. que, no obstante, les da una interpretación algo distinta de la mía:

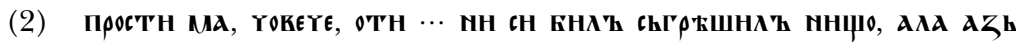

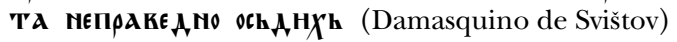

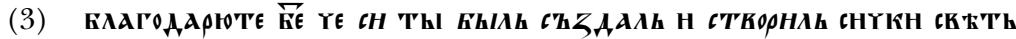
(Damasquino de Tixonravov)

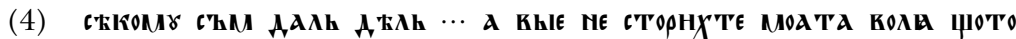

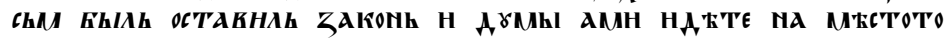
ÁE'To e naIo'TKeno Za A.IAKona. (Damasquino de Ljubljana) 
Estas formas son formal y funcionalmente equivalentes a las contemporáneas del grupo 2. Se trata de formas nuevas, no existentes en eslavo antiguo, pero que se encuentran igualmente en otros idiomas eslavos donde suponen el último eslabón de esta cadena de desarrollo. Las formas nuevas ya son fruto de la aplicación del modelo que se va a convertir en productivo en la lengua búlgara. A diferencia del resto de las lenguas eslavas, el búlgaro lo va a desarrollar más adelante y va a precisar el significado gramatical de los paradigmas de modo que nuevo y viejo puedan coexistir en el sistema de las formas verbales. Lo importante desde mi punto de vista es que no se trata de una innovación formal ajena a las lenguas eslavas, sino de un aprovechamiento máximo de posibilidades de desarrollo internas.

\section{El SIGNIFICADO}

Esta nueva visión sobre el arranque de los cambios pone en entredicho el significado inicial, e incluso el principal, de las formas. Como ya hemos aclarado, en la lingüística búlgara se acepta que el impulso ha llegado desde la lengua turca o, como mucho, se sanciona la influencia por parte de la lengua protobúlgara, también de origen turanio, pero siempre pensando en la misma categoría bien conocida en las lenguas turanias, de significado «renarrativo» que expresa la acción como «mediata», «retransmitida». La oposición principal se daría entre la expresión mediata y la no-mediata de la acción, entre la «retransmisión» y la «transmisión directa», y la lengua búlgara emplearía recursos morfológicos específicos para denotar los hechos conocidos mediante el testimonio de los demás, diferenciándolos de los conocidos directamente por el hablante. Obviamente, este significado gramatical caracteriza parte de las formas pero, como pretendo demostrar, se trata de una ampliación semántica y funcional secundaria, no del significado central del nuevo paradigma gramatical.

Creo que el tiempo siempre ha sido y sigue siendo la categoría gramatical dominante en el verbo búlgaro. Esta sería su gran diferencia con el resto de las lenguas eslavas, donde el aspecto ha llegado a ser predominante ${ }^{5}$. Por ello, considero que en el desarrollo y precisión del sistema temporal debemos buscar primero la explicación de los nuevos paradigmas creados en torno del perfecto. Soy partidaria de una descripción del sistema verbal búlgaro desde los presupuestos de la temporalidad. En esta descripción serían de aplicación dos grandes principios organizativos propuestos para el sistema temporal:

\footnotetext{
${ }^{5}$ Lo que no significa que podemos menospreciar el peso del aspecto en el verbo búlgaro, pero está claro que a nivel formal no se ha convertido en columna vertebral de su sistema morfológico.
} 
- el de Benveniste y Weinrich, que consiste en el establecimiento de dos grupos de formas verbales con dos planos de aplicación diferentes, o sea, de dos grupos de tiempos (el de la historia y el del discurso, según el primero, y el del mundo narrado y el del mundo comentado, según el segundo);

- el de análisis vectorial de la temporalidad de Guillermo Rojo, de interés especial en la descripción del verbo búlgaro y nuestro punto principal de apoyo en el estudio comparativo.

Comparto totalmente la opinión de Rojo 1974, p. 77, de que «la temporalidad lingüística está en estrecho paralelismo con el tiempo cronológico subjetivo», algo que la lengua búlgara corrobora de forma clara. En la misma línea de pensamiento sitúo la noción de actitud temporal que, según señala López García 1990, pp. 111 y s., define la concepción del tiempo como algo filtrado por la subjetividad del hablante.

Las preguntas que nos planteamos ahora son: ¿qué permitió que en la lengua búlgara las nuevas formas del pasado eslavas no sustituyeran al paradigma del pretérito antiguo? ¿Qué permitió a estas formas nuevas completarse y convertirse en paralelas a las antiguas?

Como podemos observar, las formas de futuro pretérito y de futuro pretérito perfecto del grupo 2 , totalmente desconocidas en el resto de los idiomas eslavos, demuestran una reproducción del arquetipo de composición que cohesiona, da transparencia formal al nuevo paradigma y lo convierte en una imagen especular del paradigma antiguo del pasado:

\begin{tabular}{llll} 
& \multicolumn{1}{c}{$\begin{array}{c}\text { Paradigma } \\
\text { antiguo del pasado }\end{array}$} & & \multicolumn{1}{c}{$\begin{array}{c}\text { Paradigma } \\
\text { nuevo del pasado }\end{array}$} \\
\cline { 2 - 2 } Aor. & pisah & & pisal săm, \\
Imperf. & pišeh & pišel săm \\
Pluscuamperf. & bjah pisal & & bil săm pisal \\
Futuro Pret. & štjah da piša & & štjal săm da piša \\
F. Pret. Perf. & štjah da săm pisal & & štjal săm da săm pisal
\end{tabular}

En el paradigma nuevo el perfecto antiguo ocupa el lugar del aoristo, el tiempo principal de pasado que marca el punto de referencia temporal para el resto de los tiempos pasados. Se posiciona así como eje de referencia, centro de su propio plano temporal de pasado. Su consolidación junto con el grupo antiguo de tiempos del pasado exigía el establecimiento de una diferencia suficiente de significado que residiría en las diferencias temporales entre el perfecto y el aoristo, y acogería también la categoría verbal turania que marca la diferencia entre formas testimoniales y no-testimoniales. Una acción pasada vista por el hablante desde fuera, no como una vivencia, puede relacionarse con facilidad 
con las formas no-testimoniales. La información indirecta sería, en un principio, solo una característica circunstancial para las nuevas formas de pasado que marcan la acción como vista a distancia por los participantes en el acto comunicativo. En este primer momento las nuevas formas emplearían todas las personas y el auxiliar seguiría siendo habitual en tercera persona.

Un paso de suma importancia sería el desdoblamiento posterior del significado. Creo que gracias a este desdoblamiento se ha llegado a diferenciar un pasado remoto (para sucesos anteriores a la época de los hablantes) de la serie de formas que marcan la acción como ajena al hablante, no-testimonial o presentada como tal. Volveremos a estos procesos más adelante y buscaremos su explicación desde la teoría vectorial de Rojo.

5. El SISTEMA TEMPORAL BÚLGARO Y EL ESPAÑOL.

LA TEORÍA VECTORIAL APLICADA AL SISTEMA VERBAL BÚLGARO

Un estudio comparativo entre el sistema temporal búlgaro y el español es del todo imprescindible tanto a nivel teórico, como a nivel didáctico.

El primer acercamiento al problema ya pone de relieve el sorprendente parecido entre los sistemas temporales de los dos idiomas. Desde el punto de vista del investigador de la lengua búlgara este parecido es, si cabe, aun más asombroso, ya que el búlgaro no muestra tal parecido con ningún idioma de su propio grupo lingüístico eslavo.

Los dos idiomas presentan un sistema básico de nueve tiempos verbales. En un primer momento me voy a centrar en los tiempos del indicativo español y en las formas de indicativo, definidas aquí como grupo 1 del sistema temporal búlgaro.

Como ya he señalado, en el estudio comparativo mi punto de apoyo principal será el análisis vectorial de la temporalidad de Rojo, desarrollado en solitario (Rojo 1974, 1990) y más adelante en colaboración con Veiga 1999, pp. 2867-2934, y que me parece de gran utilidad también en la descripción del verbo búlgaro. Partiendo de los postulados teóricos de Rojo sobre la temporalidad en el verbo español, presentaré las coincidencias e intentaré explicar ciertas diferencias entre las dos lenguas en el uso del binomio perfecto-aoristo de especial interés aquí. Creo que aplicar al verbo búlgaro la teoría vectorial permite resolver también una serie de problemas que afectan a los grupos 2 y 3 nuevos en el sistema verbal búlgaro ${ }^{6}$.

\footnotetext{
${ }^{6}$ En la comparación adoptaré el sistema de formulación vectorial de Rojo y me serviré de las siguientes abreviaturas: $\mathrm{O}$ (origen), oV (vector de simultaneidad), $-\mathrm{V}$ (vector de anterioridad), $+\mathrm{V}$ (vector de posterioridad), $\mathrm{P}$ (punto temporal cualquiera), $\mathrm{R}$ (acto de retransmisión).
} 
TABLA 1: Esquema paralelo de los tiempos y su fórmula vectorial.

\begin{tabular}{|c|c|c|}
\hline & Búlgaro & Español \\
\hline OoV Presente ${ }^{7}$ & piša & escribo \\
\hline O-V Aoristo & pisah & escribí \\
\hline $\mathrm{O}+\mathrm{V}$ Futuro & šte piša & escribiré \\
\hline$(\mathrm{OoV})-\mathrm{V}$ Perfecto & pisal săm & he escrito \\
\hline (O-V)oV Imperfecto & pišeh & escribía \\
\hline$(\mathrm{O}-\mathrm{V})-\mathrm{V}$ Pluscuamperfecto & bjah pisal & había escrito \\
\hline$(\mathrm{O}+\mathrm{V})-\mathrm{V}$ Futuro Perfecto & šte săm pisal & habré escrito \\
\hline$(\mathrm{O}-\mathrm{V})+\mathrm{V}$ Futuro Pretérito ${ }^{8}$ & štjah da piša & escribiría \\
\hline$((\mathrm{O}-\mathrm{V})+\mathrm{V})-\mathrm{V}$ Fut. Pret. Perfecto & štjah da săm pisal & habría escrito \\
\hline
\end{tabular}

Una comparación entre los usos rectos de los tiempos búlgaros y los españoles muestra que son totalmente equiparables, con ciertas limitaciones en el caso de la distribución entre perfecto y aoristo ${ }^{9}$.

En ambos idiomas las formas que indican posterioridad primaria son solo dos: $\mathrm{O}+\mathrm{V}$ escribiré / šte piša y $(\mathrm{O}-\mathrm{V})+\mathrm{V}$ escribiría / štjah da piša.

También son dos las formas de simultaneidad primaria: OoV escribo / piša y (O-V)oV escribia / pišeh.

Mucho más numeroso es el grupo de tiempos que indican anterioridad primaria. Aquí tiene realización formal la relación de anterioridad en cuanto a todos los ejes de referencia del sistema: O-V escribi / pisah;

${ }^{7}$ Por razones de unidad mantengo la denominación de los tiempos según la adoptada para el búlgaro. Hay que destacar que, en sus estudios de 1974, 1976 y 1990, Rojo se limita a las fórmulas sin decantarse por una terminología, pero en el trabajo común con Veiga 1999 acomodan a su nomenclatura las denominaciones propuestas por Bello 1947, o sea: presente, pretérito, futuro, ante-presente, co-pretérito, ante-pretérito, ante-futuro, pos-pretérito, ante-pos-pretérito. Estas denominaciones, muy apropiadas también para el verbo búlgaro, no se utilizan en este trabajo por ser en su mayoría ajenas a la tradición gramatical búlgara.

${ }^{8}$ Nótese que Bello 1847, Gili Gaya 1943 y Rojo y Veiga 1999 consideran que escribiría y habría escrito forman parte del indicativo, al contrario de otras posturas, por ejemplo RAE 1931 que define estas formas como «modo potencial». Llamadas en el Esbozo 1973 «condicional» y «condicional perfecto» estas formas no obstante están incluidas en el indicativo. Las denominaciones «pos-pretérito" y «ante-pos-pretérito» de Bello son las adoptadas por Rojo y Veiga 1999, pp. 2882- 2884, 2893, 1894, y estos autores las consideran compatibles con su sistema de formulación vectorial.

${ }^{9}$ Sobre los usos de estos tiempos en búlgaro y una comparación inicial con el uso español me he extendido en Láleva 1996, p. 83-84 y 91-92. 
(OoV)-V he escrito / pisal săm; $(\mathrm{O}+\mathrm{V})-\mathrm{V}$ habré escrito / šte săm pisal; $(\mathrm{O}-\mathrm{V})-\mathrm{V}$ habia escrito / bjah pisal; $((\mathrm{O}-\mathrm{V})+\mathrm{V})-\mathrm{V}$ habría escrito / štjah da săm pisal. En todos los casos y en ambas lenguas si la anterioridad no está directamente orientada al origen se expresa mediante una forma verbal compuesta que incluye obligatoriamente el participio. Podemos afirmar que el participio (el participio del aoristo en el caso búlgaro), es la expresión formal de anterioridad ${ }^{10}$.

El punto débil de este sistema temporal común para el búlgaro y el español es, sin duda alguna, el perfecto, y más concretamente la distinción entre el perfecto y el aoristo. Podemos observar que otros idiomas eslavos y románicos han prescindido de uno de estos tiempos y que existen dialectos en cada una de las dos lenguas comparadas que han emprendido el mismo camino $^{11}$. Como apunta Rojo 1974, p. 106: «no podemos dejar de reconocer que la delimitación entre las dos formas es lo suficientemente borrosa como para permitir que la distinción tienda a desaparecer en algunas zonas». No obstante, hoy se mantiene una diferencia nítida entre ambas formas en las dos lenguas estándar comparadas. La fórmula $(\mathrm{OoV})-\mathrm{V}$ de Rojo es sin dudas la apropiada tanto para el español, como para el búlgaro, pero esto no quiere decir que el valor de las formas coincida en detalle. Hay que pensar en una lectura más general de la fórmula que refleje lo común y permita lo diferente en el valor del pretérito perfecto en ambos idiomas. Tratando el material español, Rojo afirma que he llegado indica «anterioridad dentro de lo simultáneo al origen», y más adelante «dado que implica la simultaneidad indirecta al origen, he llegado suele indicar acciones más próximas a él que las expresadas mediante la forma llegué». En el caso del búlgaro tenemos que insistir en que no se trata de una «anterioridad dentro de lo simultáneo al origen», sino de una «anterioridad en cuanto a lo simultáneo al origen». En ambos casos se trata de «anterioridad desde una perspectiva simultánea al origen», de una «actualización de la anterioridad desde la posición del hablante» que puede concretarse de manera diferente. De esta forma se explican con facilidad los usos comunes del perfecto

- como acción pasada asociada al presente sobre todo gracias a sus resultados o consecuencias, tipo izljazăl e 'ha salido' (implica: no está aquí);

\footnotetext{
${ }^{10}$ Se trata de la voz activa. En las pasivas llamadas perifrásticas, que tienen el participio como característica propia, la anterioridad se expresa mediante un segundo participio, cf. está escrito / napisano $e$ : ha sido escrito / bilo e napisano.

11 Un estudio comparativo del problema a mayor escala en ambos grupos lingüísticos podría ser muy interesante sobre todo si incluye el nivel diacrónico que permitiría apreciar la interrelación entre las diferencias y las tendencias de desarrollo.
} 
- como acción ideal en el pasado sin precisar ni el momento, ni el acto mismo de su ejecución, y que se usa con locuciones temporales como vinagi 'siempre', dosega 'hasta ahora', nikoga 'nunca' y semejantes, tipo ošte ne săm go nameril 'aún no le he encontrado'.

y también los usos diferentes. La inclusión de la acción en una perspectiva temporal claramente centrada por el hablante en el plano del presente mediante referencias concretas como este siglo/año/día, que para el español peninsular supone el uso obligatorio del perfecto, en búlgaro no lo exige. Al revés, con estas locuciones se prefiere el aoristo: Tova ljato bjah v Sofija, 'Este verano he estado en Sofía'. Cuando aparezca con una locución de este tipo, el perfecto búlgaro no depende de ella, sino del deseo del hablante de presentar desde su perspectiva actual la acción como un hecho abstracto, no visto desde dentro, sino desde fuera. Con ello la gran diferencia entre el búlgaro y el español se da en el tratamiento de la simultaneidad secundaria del perfecto (OoV)-V como simultaneidad ampliada en español, o como simultaneidad de la valoración en búlgaro, y cada valoración presupone un distanciamiento inicial. En búlgaro esta abstracción y distanciamiento del perfecto lo hace incompatible con la expresión de una relación afectiva con el presente, justamente al revés del español donde presenta acciones psicológicamente más cercanas al hablante, cf. Kučeto mi umrja (aor.) = 'mi perro ha muerto' (perf.), frente a Kučeto mi e umrjalo (perf.) = 'mi perro murió' (aor.).

Las diferencias en el uso del perfecto que son resultado del tratamiento distinto de la simultaneidad al origen como punto de referencia permiten ver de forma diferente el desarrollo particular de las formas en $-l$ en la lengua búlgara. $\mathrm{El}$ perfecto $(\mathrm{OoV})-\mathrm{V}$ con su anterioridad primaria en cuanto a la simultaneidad al origen supone el posicionamiento del hablante no dentro de la acción referida sino fuera de esta, como observador situado en el momento de habla (OoV). Dado que el momento de habla y el origen podrían ser vistos como uno mismo, es fácil de entender las posibilidades del perfecto de sustituir al aoristo y de convertirse en el tiempo del pasado por excelencia, algo que encontramos en el resto de las lenguas eslavas. En búlgaro su conservación está relacionada con la creación de un eje de relación temporal propio $(\mathrm{OoV})-\mathrm{V}$ (con vector originario oV) alrededor del cual se crearon formas de anterioridad, simultaneidad y posterioridad primarias:

$\begin{array}{ll}(\mathrm{OoV})-\mathrm{V} & \text { pisal săm } \\ ((\mathrm{OoV})-\mathrm{V})-\mathrm{V} & \text { bil săm pisal } \\ ((\mathrm{OoV})-\mathrm{V}) \mathrm{oV} & \text { pišel săm } \\ ((\mathrm{OoV})-\mathrm{V})+\mathrm{V} & \text { štjal săm da piša } \\ (((\mathrm{OoV})-\mathrm{V}))+\mathrm{V})-\mathrm{V} & \text { štjal săm da săm pisal }\end{array}$


Paralelas a las creadas alrededor del eje O-V:

$\begin{array}{ll}\mathrm{O}-\mathrm{V} & \text { pisah } \\ (\mathrm{O}-\mathrm{V})-\mathrm{V} & \text { bjah pisal } \\ (\mathrm{O}-\mathrm{V}) \mathrm{OV} & \text { pišeh } \\ (\mathrm{O}-\mathrm{V})+\mathrm{V} & \text { štjah da piša } \\ ((\mathrm{O}-\mathrm{V})+\mathrm{V})-\mathrm{V} & \text { štjah da săm pisal }\end{array}$

Con lo cual la diferencia entre las dos series de formas reside en el eje de referencia. La diferencia entre el aoristo O-V, pasado anterior al origen, y el perfecto $\mathrm{OoV}-\mathrm{V}$, pasado visto desde la simultaneidad al origen, se extiende y gramaticaliza en la lengua búlgara gracias a la creación de la nueva serie. Como ya hemos insistido, su valor principal es temporal. Ahora vemos también a qué se debe su posibilidad de expresar la valoración del hablante.

Por su posición simultánea al origen, el hablante está distanciado del hecho referido, lo ve desde fuera. Se trata de una característica de la temporalidad de estas formas que no tiene nada que ver con la participación o no participación real del hablante en las acciones referidas. El valor temporal principal del grupo definido como 2.1 en este trabajo es de anterioridad en cuanto a la simultaneidad al origen, que conlleva la actualización en el momento de habla de una acción del plano del pasado vista desde la distancia.

El denominador común de todas las formas es la parte izquierda de sus fórmulas, ocupada por OoV-V. Si las formas pierden su relación con la simultaneidad al origen se convierten en un "pasado absoluto", pasado que no se contempla en su relación con el hablante, queda fuera de su espacio o época de existencia. El eje OoV-V pasa a ser -V manteniéndose las relaciones de posterioridad, anterioridad y simultaneidad pero en una esfera de acción diferente, fuera del trinomio yo-aqui-ahora. Esta pérdida de relación con la simultaneidad al origen se expresa en búlgaro a nivel formal con la pérdida del auxiliar en presente que afecta solo a la $3^{\underline{a}}$ persona como la menos marcada: pisal $e>$ pisal:

$\begin{array}{ll}-\mathrm{V} & \text { pisal } \\ (-\mathrm{V})-\mathrm{V} & \text { bil pisal } \\ (-\mathrm{V}) \mathrm{oV} & \text { pišel } \\ (-\mathrm{V})+\mathrm{V} & \text { štjal da piše } \\ ((-\mathrm{V})+\mathrm{V})-\mathrm{V} & \text { štjal da e pisal }\end{array}$

Así se constituye el grupo 2.2.A que abarca solo formas verbales en $3^{\mathrm{a}}$ persona. Son las formas por excelencia de los textos históricos (de historia no contemporánea), los cuentos populares y las leyendas.

Por otro lado, la falta de actualización de la acción desde la posición del hablante que conlleva no implicación, junto con el distanciamien- 
to característico del grupo 2, convierte esta serie de formas, la 2.2, en especialmente propicia para la «retransmisión». En tal caso son factibles todas las personas verbales. No obstante, y como es de esperar, la única con pérdida de auxiliar es la $3^{\underline{a}}$. Incluso cuando las formas se usan para referir acciones por medio de una información ajena, la relación no deja de ser temporal, ya que la emisión originaria será por descartado anterior $-\mathrm{V}$ al acto de retransmisión $\mathrm{R}$, que es simultáneo al origen $\mathrm{OoV}$.

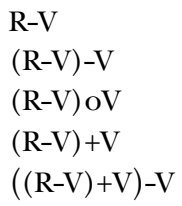

\author{
pisal săm, pisal \\ bil săm pisal, bil pisal \\ pišel săm, pišel \\ štjal săm da piša, štjal da piše \\ štjal săm da săm pisal, štjal da e pisal
}

Son las formas del grupo 2.2.B. Su eje temporal R-V sitúa las relaciones temporales primarias en relación con el momento de la emisión originaria (R-V). Así la relación de la acción retransmitida con el origen no es directa, sino que se realiza mediante la retransmisión (R) cuyo vector es simultáneo al origen OoV. En este acto el hablante retransmisor se sitúa como no implicado en la acción retransmitida, ya que no tiene una relación directa con esta, lo que se materializa en las formas sin auxiliar en $3^{\underline{a}}$ persona. La teoría vectorial permite ver desde una perspectiva nueva la «renarración» en la lengua búlgara. El uso recto de las formas que señalan la retransmisión no supone otro matiz adicional no temporal. Se trata, por lo tanto, de una ampliación de significado de las formas temporales a base de su valor principal con punto axial OoV-V, algo que confirma nuestra hipótesis de partida, según la cual la temporalidad verbal es el eje organizador principal del sistema verbal búlgaro, y explica desde otro ángulo su posibilidad de asumir en su significado la retransmisión. Así se aclara también el hecho de que el uso renarrativo se distingue dentro del temporal únicamente mediante el contexto, si este lo es lo suficientemente claro. No son formas propiamente «renarrativas». Mientras en la $3^{\underline{a}}$ persona la distinción contextual es más fácil, ya que depende del tipo textual, en la $1^{\underline{a}}$ y en la $2^{\underline{a}}$ encontramos una gran cantidad de casos cuyo significado queda abierto sin un contexto apropiado, véase por ejemplo:

(5) Izglexda, če po tova vreme ne si znael ispanski.

Parece que por aquel entonces no sabías español. Uso «conclusivo».

(6) Uveriha me, če po tova vreme ne si znael ispanski.

Me aseguraron que por aquel entonces no sabías español. Uso «renarrativo».

(7) Ne si znael ispanski.

Sin contexto tiene una lectura doble. 
Considero que la «renarratividad» en búlgaro parte y sigue siendo una parte del empleo de un grupo de formas de su sistema temporal. Desde un punto de vista comparativo, esto significa que, dado que el español no dispone del eje OoV-V, su única correspondencia posible serían los tiempos con eje O-V. La traducibilidad de las formas búlgaras se aseguraría mediante las formas temporales españolas correspondientes a las búlgaras del grupo 1 y paralelas al grupo 2 .

Para el ejemplo anterior la fórmula sería:

$$
((\mathrm{OoV})-\mathrm{V}) \mathrm{oV} \text {...si znael } \quad>\quad(\mathrm{O}-\mathrm{V}) \mathrm{oV} \text { sabias }
$$

División interna del nuevo grupo 2 en el plano del pasado

Todas las formas del grupo 2 en uso recto ocupan el plano del pasado, pero este pasado se ve desde una perspectiva de alejamiento. Si parafraseamos la terminología de Weinrich 1968, este sería en búlgaro el mundo narrado, mientras las formas del «indicativo tradicional» pertenecerían al «mundo participado» que tendría dos parcelas, la del «mundo comentado» en el plano actual, y la del «mundo rememorado» en el plano de la memoria cubierto por los tiempos del pasado tradicional.

TABLA 2: Esquema de la distribución.

\begin{tabular}{|c|c|c|}
\hline \multicolumn{3}{|c|}{$\begin{array}{c}\text { Grupo } 2 . \\
\text { Pasado ajeno a la memoria }^{12}\end{array}$} \\
\hline \multicolumn{2}{|c|}{$\begin{array}{l}\text { - implicación personal: } \\
\text { - auxiliar en } 3^{\underline{a}} \text { persona }\end{array}$} & $\begin{array}{l}\text { + implicación personal: } \\
\text { + auxiliar en } 3^{\text {a }} \text { persona }\end{array}$ \\
\hline $\begin{array}{c}\text { 2.2.A } \\
\text { No coetáneo (remoto): } \\
\text { solo } 3^{\underline{a}} \text { persona }\end{array}$ & $\begin{array}{c}\text { 2.2.B } \\
\text { Coetáneo: } \\
\text { todas las personas }\end{array}$ & $\begin{array}{c}2.1 \\
\text { Todas las personas }\end{array}$ \\
\hline pišel & $\begin{array}{l}\text { pišel săm } \\
\text { pišel si } \\
\text { pišel }\end{array}$ & $\begin{array}{l}\text { pišel săm } \\
\text { pišel si } \\
\text { pišel e }\end{array}$ \\
\hline
\end{tabular}

El hablante búlgaro sitúa los sucesos del mundo no solo en relación con el momento comunicativo, sino también con un momento de suma actualidad subjetiva, el de su propia existencia, cortando el plano tem-

\footnotetext{
12 Se trata obviamente de una terminología provisional. En este momento me interesa la caracterización y la definición previa de los conceptos.
} 
poral en dos grandes bloques: antes de mi existencia y en mi propia época. El grupo 2.2.A establece una verdadera diferencia temporal con las formas antiguas. Las formas nuevas se convierten en tiempo pasado no coetáneo de los participantes en el acto comunicativo. Este tiempo, objetivamente no participado, se vincula de forma estable con la $3^{\underline{a}}$ persona ya que, lógicamente, no puede incluir a los participantes en el acto comunicativo. Son prácticamente inexistentes los estudios sobre el uso del «renarrativo» en función de pasado en los textos búlgaros. Aun así, la preponderancia absoluta de la $3^{\text {a }}$ persona es fácil de $\operatorname{apreciar}^{13}$. El fenómeno de la restricción del grupo temporal de la «historia» a la tercera persona fue postulado ya por Émile Benveniste 1959, quien lo estudia en los textos escritos del francés y lo relaciona con el passé simple. El mismo autor señala en otro trabajo (Benveniste 1969) la existencia en algunas lenguas de formas diferenciadas para designar la anterioridad más remota y la más cercana con el ejemplo del chinook, que presenta formas distintas para pasado mítico, pasado lejano y pasado reciente. Como vemos, no hace falta ir tan lejos: la descripción del búlgaro, lengua indoeuropea y presuntamente bien conocida, muestra una diferenciación formal en el plano del pasado de características parecidas.

En búlgaro la pérdida del auxiliar es el rasgo formal característico de este pasado no coetáneo, que pierde así la vinculación formal que el presente del verbo existencial le proporcionaba con la época de los interlocutores. Es de uso obligatorio y se encuentra en distribución complementaria con las formas del pasado antiguas que ocupan el plano de la memoria ${ }^{14}$. Podemos decir que, salvadas las restricciones de aplicación (solo para el pasado no coetáneo), este es el pasado búlgaro «más eslavo», ya que formalmente se corresponde con los pasados de la mayoría de los idiomas eslavos.

La diferencia formal parcial entre el grupo 2.2.B y el grupo 2.1 está en relación directa con sus diferencias semánticas. El primero sirve para la retransmisión de información ajena con pérdida del auxiliar en $3^{\underline{a}}$ persona; con el segundo se transmite información no directa pero obtenida mediante conclusiones, generalizaciones, deducciones, etc., personales, la relación con el momento comunicativo desde el cual se realizan las

${ }^{13}$ El estudio estadístico de Ivanova 1994 -rara excepción que confirma la regla- sobre el uso del «renarrativo» en el libro Fábulas de Esopo, traducción del griego realizada por Petko Slavejov en 1852, arroja resultados incontestables en este sentido. Todas las formas «renarrativas» están en $3^{\underline{a}}$ persona, salvo una, en $2^{2}$ sg. que, como señala la investigadora, está en «aoristo renarrativo". Cabe la posibilidad de tratarse de uno de los usos típicos de cierre o de apertura del texto que obedece, como veremos más adelante, a otros principios.

${ }^{14}$ Es interesante la observación de Vaseva 1988, p. 93, de que en el género literario de las memorias las acciones pasadas hace mucho tiempo se reflejan mediante formas «renarrativas». Estas acciones no podrían haber sido observadas personalmente por el autor y están a menudo alejadas también en el espacio, mientras que lo percibido de forma personal, las memorias de la niñez, se expresan con formas directas (las del pasado antiguo). 
conjeturas se materializa con la conservación del auxiliar en todas las personas: se trata de las formas conocidas como «conclusivas».

Así el hablante búlgaro puede elegir entre varias opciones formal y semánticamente diferenciadas para la presentación de una acción pasada. La elección tendrá mayor o menor fundamento objetivo, pero siempre será justificada subjetivamente.

\section{LA DISLOCACIÓN}

Partimos de las ideas de Rojo sobre la dislocación en el sistema temporal de la lengua española. Una comparación con el búlgaro nos lleva, también en este caso, a resultados interesantes.

Como señala Rojo 1974, p. 128, la idea de la dislocación es parte de una línea de consideración del conjunto de formas verbales como un sistema:

Hay empleos que aparentemente están en contradicción con el valor temporal inicialmente atribuido. Hay en ellos discordancia entre la relación temporal de que se parte y la función con que aparecen en ciertas oraciones. Sin embargo, si se observa la totalidad del sistema en lugar de considerar las funciones aisladas, puede constatarse la existencia de ciertas tendencias bien definidas que actúan a lo largo y ancho de todo el sistema. Toda forma con valor $\mathrm{P}+\mathrm{V}$ (donde $\mathrm{P}$ es un punto cualquiera), utilizada para indicar una relación PoV, añade a la acción expresada un matiz de probabilidad. De modo semejante, una forma P-V empleada en función PoV adquiere un matiz general de irrealidad. No se trata por tanto de contradecir los valores temporales, sino de un mecanismo que actúa en el interior del sistema mediante el cual el empleo de una forma para indicar una relación temporal distinta de la que le corresponde, implica la adición de un significado extratemporal a la acción expresada según ciertas características generales (...). Los usos procedentes de la dislocación del sistema indican también una relación temporal determinada, aunque diferente de la considerada como primordial. Y precisamente gracias a esta discordancia se consigue la adición de significados o matices extratemporales.

Los dos tipos de dislocación estudiados por Rojo, en solitario o con Veiga 1999, tienen una correspondencia indiscutible en el sistema verbal búlgaro donde, como veremos, el procedimiento de dislocación se ha convertido en morfológicamente productivo. En este trabajo estudiaremos solo el tipo P-V > PoV + irrealidad.

Se trata de un principio de dislocación común en ambas lenguas y con idénticos resultados. Pero su realización concreta afecta a diferentes partes del sistema. El paralelo es, por lo tanto, a nivel sistema, y como señalan Rojo y Veiga 1999, p. 2895, el fenómeno ha sido definido por algunos autores como universal. En la lengua búlgara la dislocación 
$-\mathrm{V}>\mathrm{oV}+$ irrealidad afecta a las formas del grupo 2.2.B, que como hemos visto tienen como característica común el momento axial anterior a la retransmisión (la parte izquierda de la fórmula). Esta característica las hacía apropiadas para la expresión de la «renarratividad». El cambio del plano de pasado R-V a uno actual RoV en la función de cada una de estas formas, que siempre depende del contexto, conlleva un matiz adicional de irrealidad. Es la manera que tiene el hablante de expresar sus reservas en cuanto a la información retransmitida y presentarla como irreal desde su propio punto de vista en el momento de la retransmisión $\mathrm{R}-\mathrm{V}>\mathrm{RoV}+$ irrealidad.

Véase:

$(\mathrm{R}-\mathrm{V})+\mathrm{V}$

(8) Predložiha mi da otida s tjah. Šteli sme da posetim Belgija i Francija i da podelim razhodite za benzin, no az njamah nužnite pari i ne možah da otida.

Me ofrecieron ir con ellos. Visitaríamos Bélgica y Francia y compartiríamos los gastos de gasolina, pero yo no tenía el dinero necesario y no pude ir.

$(\mathrm{R}-\mathrm{V})+\mathrm{V}>(\mathrm{RoV})+\mathrm{V}+$ irrealidad

(9) Steli sme da posetim Belgija i Francija. Kak šte gi posetim kato njamame pari!

Visitaríamos Bélgica y Francia. ¡Cómo las vamos a visitar si no tenemos dinero!

La dislocación afecta todas las formas del grupo y aclara su doble uso, que tantos quebraderos de cabeza ha dado a los gramáticos búlgaros:

Imperfecto en función de Presente + irrealidad

$(\mathrm{R}-\mathrm{V}) \mathrm{oV} \quad>\quad(\mathrm{RoV}) \mathrm{oV}$

pišel săm, pišel

Pluscuamperfecto en función de Perfecto + irrealidad

$(\mathrm{R}-\mathrm{V})-\mathrm{V} \quad>\quad(\mathrm{RoV})-\mathrm{V}$

bil săm pisal, bil pisal

Futuro Pretérito en función de Futuro + irrealidad

$(\mathrm{R}-\mathrm{V})+\mathrm{V} \quad>\quad(\mathrm{RoV})+\mathrm{V}$

stjal săm da piša, štjal da piše

Futuro Pretérito Perfecto en función de Futuro Perfecto + irrealidad

$((\mathrm{R}-\mathrm{V})+\mathrm{V})-\mathrm{V} \quad>\quad((\mathrm{RoV})+\mathrm{V})-\mathrm{V}$

štjal săm da săm pisal, štjal da e pisal

En búlgaro, como ya hemos visto, las formas temporales nuevas se componen mediante la aplicación reiterada de modelos analíticos pre- 
vios. El procedimiento de la dislocación también se convierte en modelo y de este modo pasa a ser un procedimiento gramatical. Como tal se aplica a las formas del mismo grupo 2.2.B pero esta vez para expresar la irrealidad de la acción desde el punto de vista del hablante retransmisor, sin abandonar el plano del pasado. Se materializa mediante la ampliación de las formas con un -V por excelencia: el participio en $-l$, bil, del verbo auxiliar que, no obstante, no aporta, ni puede aportar, anterioridad adicional a las formas, sino que les confiere un matiz extratemporal de irrealidad. De esta manera se llega a las formas del grupo 2.3:

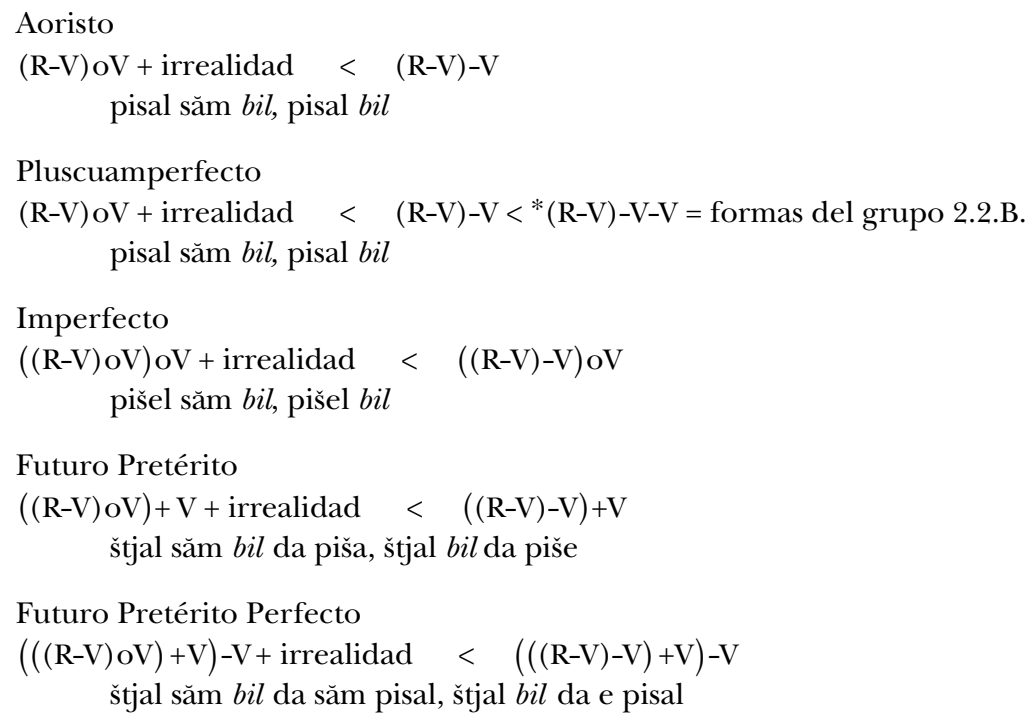

Como vemos, la ampliación afecta a todas las formas del pasado, salvo a las de pluscuamperfecto, que obedecen a las limitaciones propias del sistema temporal búlgaro, que no admite más de dos vectores de anterioridad (-V) seguidos, lo que aclara la coincidencia formal entre las formas del aoristo y del pluscuamperfecto. A nivel formal esto se refleja mediante el rechazo de un segundo бил en la forma compuesta. La dislocación deja de ser un hecho intrasistemático y se convierte en un procedimiento morfológico.

(10) Predložiha mi da otida s tjah. Šteli sme bili da posetim Belgija i Francija i da podelim razhodite za benzin.

Me ofrecieron ir con ellos. Que íbamos a visitar Bélgica y Francia y compartir los gastos de gasolina. (No me lo creí / me pareció un timo, etc.)

Fácilmente podemos apreciar que se trata de un procedimiento que la lengua española no comparte, lo que provoca la necesidad de una ampliación explicativa en la traducción. 
Creo que podemos considerar estos dos conjuntos de formas -con dislocación intrasistemática y con dislocación morfológica-como las verdaderas realizaciones a nivel funcional de una «renarratividad»con carga extratemporal en la lengua búlgara. Llevan siempre el significado no temporal de irrealidad. Nótese que se trata de dos tipos de formas, unas que alteran su valor por el cambio de posición dentro del sistema, y otras que adquieren este valor a nivel formal con un procedimiento de composición que aplica las pautas de la dislocación intrasistemática a nivel morfológico.

Podemos concluir que en un momento del desarrollo del sistema verbal búlgaro las formas del grupo 2.2.B han empezado a emplearse también en el plano actual. En principio, esta transferencia, «metáfora temporal» en términos de Weinrich, mantiene una tensión entre la significación propia (de pasado) y la determinación verdadera (en el plano actual) en el contexto. Se trata de una «dislocación» en términos de Rojo, paso de un vector $-\mathrm{V}$ a un vector $\mathrm{oV}+$ irrealidad por la dislocación del último vector $-\mathrm{V}$, el izquierdo, que es el que el participio en $-l$ aporta. El significado de irrealidad que caracteriza las formas búlgaras del grupo 2.2.B en su uso desplazado en el plano actual (presente, futuro, perfecto, futuro perfecto) se gramaticaliza y con ello también, el procedimiento $-\mathrm{V}>\mathrm{oV}$ con el cual se lleva a cabo. Se completa aplicado esta vez sobre las formas originarias del grupo con la aglutinación de un nuevo participio en $-l$ del auxiliar, que es la única forma de conseguir un desplazamiento paralelo a $-\mathrm{V}>\mathrm{oV}$ dentro del plano del pasado. Así se llega al subgrupo 2.3, el más reciente del grupo 2, caracterizado y creado a base del participio en $-l$.

TABLA 3.

\begin{tabular}{|c|c|c|}
\hline \multicolumn{3}{|c|}{ Dislocación tipo $-\mathrm{V}>\mathrm{oV}+$ irrealidad } \\
\hline \multirow[b]{2}{*}{ 2.2.B } & \multicolumn{2}{|r|}{ intrasistemática } \\
\hline & $\begin{array}{l}\text { Presente } \\
\text { Perfecto } \\
\text { Futuro } \\
\text { Futuro Perfecto }\end{array}$ & $\begin{array}{l}\text { pišel săm, pišel } \\
\text { bil săm pisal, bil pisal } \\
\text { štjal săm da piša, štjal da piše } \\
\text { štjal săm da săm pisal, štjal da e pisal }\end{array}$ \\
\hline & \multicolumn{2}{|r|}{ gramaticalizada } \\
\hline 2.3 & $\begin{array}{l}\text { Aoristo } \\
\text { Imperfecto } \\
\text { Pluscuamperfecto } \\
\text { Futuro Pretérito } \\
\text { Futuro Pretérito Perfecto }\end{array}$ & $\begin{array}{l}\text { pisal săm bil, pisal bil } \\
\text { pišel săm bil, pišel bil } \\
\text { pluscuamperfecto de grupo 2.2.B. } \\
\text { štjal săm bil da piša, štjal bil da piše } \\
\text { štjal săm bil da săm pisal, štjal bil da e pisal }\end{array}$ \\
\hline
\end{tabular}




\section{FENÓMENOS DEL USO QUE PODEMOS EXPLICAR}

Este nuevo acercamiento al problema estudiado puede explicar ciertos fenómenos de uso de las formas verbales búlgaras de los distintos grupos que hasta ahora no han sido tratados, o no han encontrado un tratamiento adecuado en la gramática de la lengua búlgara. Entre estos quiero destacar por su importancia:

1. El uso desordenado en canciones populares de los pretéritos del grupo 1 y del grupo 2 (2.1 y 2.2).

2. El cambio del grupo 1 al grupo 2 en el estilo indirecto que afecta únicamente los tiempos del pasado.

3. El uso de formas, sobre todo la del aoristo, del grupo 2.1 en frases introductorias (¿y de cierre?) de narraciones que se realizan de forma obligatoria en los tiempos del grupo 2.2.A (textos históricos, leyendas, etcétera).

4. La ampliación contemporánea del uso de las formas del grupo 2.1 a expensas de las del grupo 2.2.A.

Me detendré brevemente en cada uno de estos fenómenos.

1. En la gramática búlgara (cf., por ejemplo, Stojanov 1983, p. 364, en la gramática de la Academia de Ciencias de Bulgaria), se considera que el uso desordenado -sin diferencias en el significado- de los tiempos de pasado tradicionales y las formas «renarrativas» en la canción popular se debe ¡a razones de ritmo!, ya que las formas «renarrativas», como más tardías, no habrían conseguido sustituir las anteriores sin afectar la estructura del verso. Una explicación de este tipo me parece muy difícil de sostener. Voy a demostrar que se trata de una reminiscencia del primer período de coexistencia de las formas nuevas y las formas antiguas de los tiempos del pasado. Registrada por los folcloristas búlgaros a mediados del siglo xix, ya estaba entonces en vías de desaparición. Lo que tenemos que averiguar es si hoy se conserva de forma efectiva, lo que estaría en relación con su posibilidad de obtener funciones estilísticas como una de las señas de identidad de la canción popular búlgara. Stojanov 1983 aporta como ejemplos versos de dos canciones con mezcla de formas indicativas y «renarrativas» (o sea, del grupo 2.2) y en una nota al final del apartado apunta también la posibilidad de encontrar en la canción popular, junto con las anteriores, también la forma del pretérito perfecto en uso «renarrativo», con el ejemplo: Vljazla e v malka gradinka, / nabrala cvete vsjakakvo, / nakiti kitka šarena. Como se puede apreciar, se trata de un aoristo del grupo 2.1 [+implicación del hablante, + conservación del auxiliar]. En realidad, en un primer momento podemos tratar las formas con y sin auxiliar como grupo úni- 
co, ya que en el período de coexistencia, con el cual relacionamos el fenómeno, el uso del auxiliar en las formas del pasado nuevas es opcional. Mis primeras comprobaciones se centraron en dos colecciones de textos procedentes de finales del siglo XIX, las canciones recogidas en el Diccionario de la Lengua Búlgara de N. Gerov 1895-1904, y las publicadas como anexo en el Obzor Zvukovy " i formal'nyh" osobennostej bolgarskogo jazyka de P. Lavrov 1893. El Diccionario de Najden Gerov es un léxico preparado a mediados del siglo XIX, mediante recogida directa del material en el habla popular. Como subraya el autor (Gerov 1975, t. I, p. IV):

(...) Mi intención desde siempre ha sido presentar en mi diccionario la lengua búlgara tal como está en el pueblo y, de las palabras extranjeras, solo aquellas que emplea el pueblo. Por ello, de los libros que tenemos ahora no he cogido ni una sola palabra, ni una frase como ejemplo, y todo lo que tengo puesto en el diccionario, lo tengo anotado de la boca del pueblo y del folclore popular: paremias, canciones, etc., para poder decir que publico un diccionario de la lengua búlgara popular viva y dejar así un testimonio del que se podrá apreciar cómo ha sido la lengua búlgara hablada por el pueblo a los albores de la literatura búlgara moderna.

Por tanto se trata de textos del todo auténticos, no influenciados por la lengua literaria, ni redactados al antojo del recopilador. La situación que reflejan es justamente la que esperaríamos para el primer período de coexistencia entre el nuevo y el viejo en los tiempos del pasado. Dentro de los ejemplos encontrados en el tomo II del Diccionario cabe destacar unos cuantos muy ilustrativos:

I.

Sam"-si Stojan sja izlăga,

Če i rokljă-tă извади,

I Marijka sja razvr'-tě,

Če iz' komina izvr'knă,

Če na kăštă- tă kacnăla.

II.

Naj-podir" Radka ostană,

Să lěvă e răkă hvărlila,

Kuršum' prěz" pr'sten prěmină,

Tja si vojvoda stanăla;

Vsičky-ti klon" ostanăli,

Če tja vojvoda da stane.

III.

Natoči mu edin" čebur",

Edin čebur", zojno vino,

Ka go dignă Grue dětja,

Na dušek" go vse izpilo. 
Como se puede apreciar, el uso de los tiempos del pasado en estos ejemplos -como en un número muy significativo de textos de canciones populares aportados por el Diccionario- no sigue ninguna de las reglas que actúan sobre su distribución actualmente. No obstante, esto no es una constante y se pueden encontrar también varios ejemplos que ya traslucen el camino de desarrollo de las formas. Un buen ejemplo -más ilustrativo dado que se trata de un texto de canción completo- del uso de los tiempos del grupo 2 como tiempos del pasado a expensas del pasado tradicional lo encontramos en Lavrov 1893. A diferencia de Gerov, quien no aporta datos sobre la procedencia regional de las canciones, los textos de Lavrov proceden todos de la región de Pirdop, recogidos por T. Vlajkov, otro buen conocedor del folclore búlgaro.

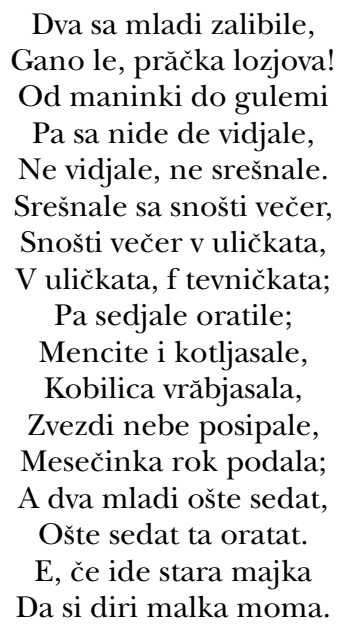

En esta canción el pasado está completamente ocupado por las formas nuevas, ¡todas en $3^{\underline{a}}$ persona y sin auxiliar! La actualización desde el momento comunicativo se realiza mediante el cierre en presente de los últimos cuatro versos. Me parece significativo que la situación que refleja esta canción está en plena concordancia con lo que encontraríamos en cualquier cuento popular, o sea, tiempos del grupo 2.2.A. En los textos de Lavrov, de la misma época que los de Guerov, en realidad no encontramos el famoso «uso desordenado» de la canción popular. Por el contrario, las canciones muestran uno u otro de los grupos de tiempos, pero nunca su mezcla. Un futuro estudio del problema debe tener en cuenta la procedencia regional de los textos ya que, al parecer, el desarrollo no se ha producido con la misma velocidad y nos podemos encontrar también con diferencias estilísticas típicas de una región y desconocidas en otras. 
2. El cambio del grupo 1 al grupo 2 en el estilo indirecto búlgaro afecta solo a los tiempos del pasado. Hay que tener en cuenta que en búlgaro la correlación temporal no afecta a las subordinadas indirectas. Estas conservan el tiempo verbal de la oración directa independientemente del tiempo verbal de la oración principal. Llama la atención el hecho de que el aoristo y el pretérito imperfecto no conservan las formas del grupo 1 en estilo indirecto sino que las sustituyen con las formas correspondientes del grupo 2, el del pasado ajeno a la memoria del hablante. La relación entre la conservación del auxiliar y la implicación personal daría respuesta a la fluctuación entre formas del grupo 2.1 y del grupo 2.2 en estos casos. El hecho está recogido en alguna de las gramáticas, pero no ha merecido explicación de ningún tipo. Esta sustitución se puede esperar para todos los tiempos del plano del pasado. La encontramos también en pluscuamperfecto, en futuro pretérito y en futuro pretérito perfecto, porque el estilo indirecto es de hecho un procedimiento típico de enajenación de la acción. Dada la menor frecuencia de estos tiempos, sobre todo de los últimos dos, y la dificultad de encontrar ejemplos en los textos literarios, este problema no ha sido tratado hasta ahora. Para obtener más datos sobre el funcionamiento de los tiempos del plano de pasado en el estilo indirecto conviene salir de la lengua escrita y estudiar también el idioma hablado.

3. El uso de formas, sobre todo la del aoristo, del grupo 2.1 en frases introductorias (¿y de cierre?) de narraciones que se realizan de forma habitual en los tiempos del grupo 2.2.A (textos históricos, cuentos populares, leyendas, etc.). Es un hecho conocido que los investigadores mencionan de paso, pero no existe unanimidad sobre el valor gramatical que se le confiere. Algunos consideran que se trata de un uso especial del perfecto del grupo 1 en el comienzo de textos o unidades textuales mayores, escritos en tiempos «renarrativos»; otros insisten en que se trata de formas «conclusivas» que vienen a introducir los hechos como deducciones, generalizaciones o conclusiones del autor. Creo que un estudio de los géneros que tienen como característica propia el uso de los tiempos del grupo 2.2.A utilizando textos completos o por lo menos unidades de texto lo suficientemente grandes, mostraría un paralelismo interesante entre el uso búlgaro y el de otras lenguas indoeuropeas. En palabras de Weinrich 1968, pp. 206 y s.:

(...) La introducción y la conclusión no son simplemente la primera y la última oración del relato, sino partes del relato que narrativamente conllevan funciones especiales. La introducción es exposición; da a conocer el mundo que va a narrarse invitando al lector o al oyente a encaminarse a él. La conclusión cierra este mundo misterioso del relato (...). De este mundo maravilloso volvemos al nuestro cotidiano (...). Se trata de dos funciones que cualitativamente son distintas del mero narrar porque marcan la divisoria 
entre el mundo comentado y el mundo narrado. Son dos funciones que circundan el propio cuerpo narrativo en el que se desarrolla el relato (...). Las tres fases de la narración, introducción, núcleo narrativo y conclusión son a la vez tres fases de los tiempos. De este modo la narración cobra relieve y se distribuye en un primer plano y en un segundo plano.

Vista desde esta perspectiva, la distribución de los grupos temporales en los textos históricos búlgaros arroja un significado distinto. Por la conservación del auxiliar (¡siempre en presente!), los tiempos del grupo 2.1 ofrecen una perspectiva desde el plano actual actualizada mediante la implicación del hablante sobre los hechos objeto de la narración nuclear. Es muy probable que los encontremos no solo en la introducción, sino también en el cierre del relato. El cierre de los cuentos populares con frases tipo $i$ az bjah tam, jadoh pih $i$ se veselih etc., en tiempos pasados tradicionales (grupo 1), que es su única aparición posible en el cuento, sería otro paso en la misma dirección, ya que asegura la vuelta definitiva a la perspectiva de la participación, a la realidad del hablante.

4. La ampliación contemporánea del uso de las formas del grupo 2.1 a expensas de las del grupo 2.2.A es un fenómeno real en desarrollo que afecta sobre todo los textos científicos y sociales, algo mencionado ya por Stankov 1967, p. 344. Aunque no comparto su explicación, reconozco la agudeza de la observación, aun más si pensamos que data del año 1967.

Según lo expuesto en el presente trabajo, los tiempos de pasado no coetáneo que se sustituyen (el grupo 2.2.A) son totalmente objetivos; senalan acciones que quedan fuera de la época de existencia del hablante; la «retransmisión» de información ajena no tiene ningún peso en su semántica. La objetividad de las formas, su independencia del hablante y del plano actual, se expresa a nivel formal con la pérdida del auxiliar en presente. Con ello, la recuperación del auxiliar -y en realidad de eso se trata-implica al hablante y el plano actual y de ningún modo puede contribuir a una mayor objetividad. Entonces, ¿qué la provoca? Creo que no se trata de una búsqueda de mayor objetividad, sino justo lo contrario. Hay que insistir en las parcelas de la vida en las que están en uso los tiempos del grupo 2.2.A. Por un lado tenemos los cuentos populares. Con estos las formas 2.2.A entraban a formar parte de la gramática del hablante desde sus primeros años de vida, primero de forma pasiva y después activa, favorecidas emocionalmente por estar relacionadas con el ocio, el trato preferente con los familiares adultos, y por ser portadoras de relatos que abrían ante el niño la puerta mágica a un mundo emocionante y atractivo. Las leyendas que la familia compartía en los 
momentos más entrañables de la jornada mantenían y reforzaban el uso de las formas en cuestión. Las obras de historia y las investigaciones históricas en la lengua búlgara moderna adoptaron de forma natural el uso de los tiempos de pasado no coetáneo (2.2.A) de las leyendas y los cuentos populares. Pero en la vida moderna se cuentan cada vez menos cuentos y las últimas generaciones ya no descubren el mundo a través del cuento. Tienen la televisión, y la imagen televisiva es actual, está reñida con los tiempos del pasado no coetáneo. El desarrollo social, un factor importante en el cambio lingüístico, lleva a un cambio generacional en el aprendizaje del idioma y de allí a una percepción diferente de las formas que nos interesan. Cuando el niño a edad escolar se encuentra con los textos históricos no relaciona las formas con pérdida de auxiliar que los caracterizan con el pasado del cuento y de la leyenda, sino con el uso renarrativo, el del grupo 2.2.B, que ya conoce bien por su gran frecuencia en el idioma hablado. Esta relación, secundaria desde un punto de vista histórico, parece estar reforzándose en la gramática del hablante en las últimas décadas. Las formas con auxiliar recuperado se ven como marca del compromiso personal con la veracidad de lo expuesto. Por ello son tan agresivas en textos y exposiciones científicas y en todo tipo de exposiciones que pretenden demostrar la convicción (siempre subjetiva) del hablante de la realidad objetiva de los hechos. La implicación del hablante convierte las formas del grupo 2.1 en las más apropiadas para un relato de hechos que cuenta con su compromiso.

\section{REFERENCIAS BIBLIOGRÁFICAS}

Aronson, H. (1967): «The grammatical categories of the indicative in the contemporary Bulgarian literary language», To Honor Roman Jakobson, La HayaParís, Mouton, pp. 82-98.

Bello, A. (1847): Gramática de la lengua castellana destinada al uso de los americanos, Santiago de Chile, Imprenta del Progreso.

Benveniste, E. (1959): «Les relations de temps dans le verbe français», Bulletin de la Société de Linguistique de Paris 54, pp. 69-82.

Benveniste, E. (1969): «El lenguaje y la experiencia humana», en Problemas del lenguaje, Buenos Aires, Ed. Sudamericana, pp. 3-12.

EsBozo (1973): Esbozo de una nueva gramática de la lengua española, Madrid, RAE.

GERDŽIKOv, G. (1973): «Bălgarskite glagolni vremena kato sistema», Izvestija na Instituta za bălgarski ezik, XXII, pp. 125-150.

- (2003): Preizkazvaneto v bălgarskija ezik, Sofia, Editorial Universitaria „Sv. Kliment Ohridski”.

Gerov, N. (1975): Rečnik na bălgarskija ezik, Bălgarski pisatel, Sofia (primera edición 1895-1904).

GiLi GaYA, S. (1943): Curso superior de sintaxis española, México, Minerva. 
Ivanov, V. (1983): Istoričeskaja grammatika russkogo jasyka, Moskva, Prosveščenie.

Ivanova, R. (1994): «Ustanovjavane na formite za preizkazvane v sistemata na novobălgarskija knižoven ezuk », Lingvistični studii, Veliko Tărnovo, „Sv.Sv. Kiril i Metodij”, pp. 311-318.

KuCAROV, I. (1979): «Gramatičeskata kategorija preizkaznost / nepreizkaznost v săvremennija bălgarski ezik», Godišnik na Sofijskija universitet, Fakultet po slavjanski filologii 70, 1, София.

— (1999): «Morfologija»v Bojadžiev, T., Kucarov, I. i Penčev, J., Săvremenen bălgarski ezik, Sofija, Izd.. Petăr Beron, pp. 277-497.

LÁleva, T. (1996): Lengua búlgara. Gramática básica, Madrid, Orto.

LAVRov, P. (1893): Obzor zvukovyh" i formal'nyh" osobennostej bolgarskogo jazyka, Moskva, Universitetskaja tipografija.

López GARCíA, A. (1990): «La interpretación metalingüística de los tiempos, modos y aspectos del verbo español: ensayo de fundamentación», en Bosque, I. (ed.), Tiempo y aspecto en español, Madrid, Cátedra.

PAŠOv, P. (1965): «Bălgarskite glagolni vremena», Narodna prosveta, 3, pp. 52-63.

— (1999): Bălgarska gramatika, Sofija, IK «Hermes».

PENČEv, J. (1967): «Kăm văprosa za vremenata v săvremennija bălgarski ezik», Bălgarski ezik, 2, pp. 131-143.

RAE (1931): Gramática de la lengua española, Madrid, RAE.

RoJo, G. (1974): «La temporalidad verbal en español», Verba 1, pp. 68-149.

— (1976): «La correlación temporal», Verba 3, pp. 65-89.

- (1990): «Relaciones entre temporalidad y aspecto en el verbo español» en Bosque, I. (ed.), Tiempo y aspecto en español, Madrid, Cátedra, pp. 17-43.

— y Veiga, A. (1999): «El Tiempo verbal. Los tiempos simples» en Bosque, I. y Demonte, V. (dir.), Gramática Descriptiva de la Lengua Española, T. 1-3, Madrid, Espasa, pp. 2867-2934.

STANKov, V. (1967): «Kategorija na indikativa v săvremennija bălgarski ezik», Bălgarski ezik, 4, pp. 330-344.

Stojanov, ST. (coord.) (1983): Gramatika na săvremennija bălgarski knižoven ezik. TII Morfologija, Sofija, Izdatelstvo na BAN.

VASEva, Iv. (1988): «Preizkazvaneto kato stilističen pohvat», Slavističen sbornik, Sofija, BAN, pp. 91-100.

WeINRICH, H. (1968): Estructura y función de los tiempos en el lenguaje, versión española de F. Latorre, Madrid, Gredos.

ZLATANOvA, P. (1986): «Istoričeski razvoj na bălgarskija ezik», Uvod v izučavaneto na južnoslavjanskite ezici, Sofia, BAN, pp. 134-215. 
